\title{
Withaferin A suppresses skin tumor promotion by inhibiting proteasome-dependent isocitrate dehydrogenase 1 degradation
}

\author{
Kaiyue $\mathrm{Xu}^{1 \#}$, Chunjing Zhang ${ }^{2 \#}$, Youbo $\mathrm{Li}^{1}$, Xin $\mathrm{Xi}^{3}$, Lishuang Zheng ${ }^{3}$, Ming Meng ${ }^{1}$, Tongtong Liu ${ }^{4}$, \\ Yunfeng Zhao ${ }^{5}$, Wenjuan $\mathrm{Li}^{1,6}$ \\ ${ }^{1}$ College of Medicine, Hebei University, Baoding 071000, China; ${ }^{2}$ Department of Biochemistry and Molecular Biology, Qiqihar Medical University, \\ Qiqihar 161006, China; ${ }^{3}$ Hebei Key Laboratory of Cancer Radiotherapy and Chemotherapy, Baoding 071000, China; ${ }^{4}$ Department of Neurology, \\ The Affiliated Hospital of Hebei University, Baoding 071000, China; ${ }^{5}$ Department of Pharmacology, Toxicology \& Neuroscience, LSU Health \\ Sciences Center in Shreveport, Shreveport, LA, USA; ${ }^{6}$ Key Laboratory of Pathogenesis Mechanism and Control of Inflammatory-autoimmune \\ Diseases in Hebei Province, Hebei University, Baoding 071000, China \\ Contributions: (I) Conception and design: C Zhang, W Li, Y Zhao; (II) Administrative support: W Li; (III) Provision of study materials or patients: W \\ Li, Y Zhao; (IV) Collection and assembly of data: K Xu, Y Li, X Xi, L Zheng, T Liu; (V) Data analysis and interpretation: K Xu, C Zhang, M Meng, \\ W Li; (VI) Manuscript writing: All authors; (VII) Final approval of manuscript: All authors. \\ \#These authors contributed equally to this work. \\ Correspondence to: Associate Prof. Yunfeng Zhao. Department of Pharmacology, Toxicology \& Neuroscience, LSU Health Sciences Center in \\ Shreveport, Shreveport, LA 33932, USA. Email: yzhao1@1suhsc.edu; Associate Prof. Wenjuan Li. College of Medicine, Key Laboratory of \\ Pathogenesis Mechanism and Control of Inflammatory-autoimmune Diseases in Hebei Province, Hebei University, 342 Yuhua East Road, Lianchi \\ District, Baoding 071000, China. Email: liwenjuan@hbu.edu.cn.
}

Background: The metabolic enzyme isocitrate dehydrogenase 1 (IDH1) belonging to $\beta$-decarboxylase dehydrogenase family has been identified as a tumor suppressor. Withaferin A (WA), a bioactive compound derived from Withania somnifera, has the anti-tumor activity. Based on the data set that WA inhibited 12-O-tetradecanoylphorbol-13-acetate (TPA)-induced IDH1 inactivation and mitochondrial dysfunction, we focused on how WA suppressed the skin carcinogenesis mediated by IDH1.

Methods: The mRNA levels of IDH1 were measured after treated with TPA and/or WA. The expression of IDH1, lactate dehydrogenase $(\mathrm{LDH})$ involved in glycolysis, hypoxia inducible factor-1 $\alpha(\mathrm{HIF}-1 \alpha)$ and its target gene glucose transporter-1 (Glut1) were detected. The activities of proteasome and the mitochondrial complex I related to mitochondrial functions were determined. The enzymatic activities of LDH, proline hydroxylase (PHD) and vascular endothelial growth factor (VEGF) were analyzed.

Results: The qPCR data have shown the mRNA levels of IDH1 were no difference with TPA and/or WA treatment. Next, data demonstrated that WA could stabilize IDH1 by inhibiting the ubiquitin-proteasome pathway (UPP). Followed by illuminating the mechanism of IDH1 inhibiting tumorigenesis, the results mirrored that upregulated IDH1 suppressed LDH activity whereas increased mitochondrial complex I activity. Furthermore, via its product $\alpha-\mathrm{KG}$, upregulated IDH1 activated PHD, and inhibited HIF-1 $\alpha$ and its downstream signaling pathway.

Conclusions: Our results indicate that WA inhibits tumor promotion partially via stabilizing IDH1, leading to inactivating the HIF-1 $\alpha$ signaling.

Keywords: Withaferin A (WA); skin carcinogenesis; ubiquitin-proteasome pathway (UPP); isocitrate dehydrogenase 1 (IDH1); chemoprevention

Submitted Feb 18, 2019. Accepted for publication Aug 28, 2019.

doi: $10.21037 /$ tcr.2019.09.57

View this article at: http://dx.doi.org/10.21037/tcr.2019.09.57 


\section{Introduction}

A metabolic shift often accompanies carcinogenesis. Cancer cells lean to utilizing a high rate of glycolysis to generate energy instead of oxidative phosphorylation (OXPHOS) even in the presence of oxygen, known as the "Warburg Effect" (1) or aerobic glycolysis. An important benefit of enhanced aerobic glycolysis is that the largely produced metabolic intermediates serve as the building blocks for biosynthesis (2). Associated with the metabolic switch is the dysregulation of crucial metabolic enzymes. Isocitrate dehydrogenases (IDHs) as the most pivotal metabolic enzyme catalyze the nicotinamide adenine dinucleotide $\left(\mathrm{NAD}^{+}\right)$-dependent or nicotinamide adenine dinucleotide phosphate $\left(\mathrm{NADP}^{+}\right)$-dependent transformation of isocitrate to $\alpha$-ketoglutarate (3). IDHs possess three isoforms: IDH3 is the main form of IDH involved in the TCA cycle, and no mutation in any of the IDH3 subunits is reported in cancer cells at present. IDH1 and IDH2 mutations have been reported in gliomas and acute myelogenous leukemia (4). Mutant IDHs produce 2-hydroxyglutarate. It is possible to regulate the hypermethylation of RIP3 promotor mediated by DNA methyltransferase (DNMT1), impairing necroptosis to contribute to tumorigenesis (5); therefore, 2-hydroxyglutarate has been recognized as an onco-metabolite (6). Unlike IDH2/3, IDH1 located in cytosol, whose substrate and product could shuttle between mitochondria and cytosol, has been determined as a tumor suppressor since its stability plays an essential role in carcinogenesis (7). Our previous study demonstrated that the protein and activity levels of IDH1 were inhibited by tumor promoter treatment, not IDH2. Therefore, maintaining the expression and activity levels of IDH1 would be of importance to carcinogenesis inhibiting.

As one of the major bioactive compounds derived from Withania somnifera, Withaferin A (WA) has been used for the treatment of nervous and sexual disorders (8). Recent studies have demonstrated the anti-tumor potential of WA, which shows that WA exerts the anti-proliferation activity in non-small cell lung cancers (9), osteosarcoma (10), breast cancer (11), prostate cancer (12), colon cancer (13), and pancreatic cancer (14), either in vitro or in vivo. Meanwhile, a clinical trial of WA has been launched for treatment of metastatic melanoma (15). Our early studies have shown that WA inhibits skin cell transformation and blocks tumor promoter-induced metabolic alterations (16), which make WA a potential chemopreventive agent. However, the molecular mechanisms of chemoprevention could be different from its anti-tumor activities.

Increasing evidences show that ubiquitin-proteasome pathway (UPP) inducing degradation of tumor suppressors plays a contributing role during carcinogenesis $(17,18)$. In detail, to cooperate with ubiquitin-activating enzymes E1 and ubiquitin-conjugating enzymes E2, ubiquitin E3 ligases combine and modify specific substrates leading to their degradation by $26 \mathrm{~S}$ proteasome (19). This ubiquitin E3 ligase pathway is crucial during tumorigenesis $(20,21)$, which may also serve as the target for chemoprevention. Based on computer simulation (22) and experimental data $(17,23)$, it has been proved that WA could bind to the 20S, 26S proteasome core complex, to inhibit the UPP. In present study, we will examine whether WA may target UPP, thereby stabilize IDH1 as an important mechanism of chemoprevention.

\section{Methods}

\section{Cell lines, reagents, and treatment}

Murine skin epidermal JB6 Cl-41 P+ cells (purchased from American Type Culture Collection) were grown in EMEM (Gibco) medium containing 5\% fetal bovine serum (FBS), penicillin and streptomycin in a $37{ }^{\circ} \mathrm{C}$ incubator under $5 \% \mathrm{CO}_{2}$. WA, dissolved in dimethyl sulfoxide (DMSO; Sigma, St Louis, MO, USA), was purchased from ChromaDex (ASB-00023250; Irvine, CA, USA). 12-O-tetradecanoylphorbol-13-acetate (TPA; P1585, Sigma), MG-132 (HY-13259; MCE, Monmouth Junction, NJ, USA), and cycloheximide (CHX; C7698, Sigma) were dissolved in DMSO.

\section{Isolation of total RNA and real-time PCR}

Total RNA was isolated from cells using E.Z.N.A total RNA kit II (OMEGA, Norcross, GA, USA) following the manufacturer's instruction. Two $\mu \mathrm{g}$ of the purified RNA was reverse transcribed using HiScript II First Strand cDNA Synthesis kit (Vazyme, Nanjing, China) according to the manufacturer's instruction. The mRNA level of IDH1 was determined by quantitative real-time PCR analysis using qPCR SYBR Green Master mix (Vazyme) which was normalized to 18S RNA as the internal control. IDH1 primer: GACTCAGTCGCCCAAGGT (Sense), GCAGCCTCTGCTTCTACC (Antisense); Nrf2 primer: TGGACGGGACTATTGAAGGCTG (Sense), GCCGCCTTTTCAGTAGATGGAGG (Antisense); 18S 
RNA primer: CTCAACACGGGAAACCTCAC (Sense), CGCTCCACCAACTAAGAACG (Antisense). Each sample was analyzed in triplicate, and the expression levels were normalized using the $2^{-\Delta \Delta \mathrm{Ct}}$-based fold change method.

\section{Preparation of whole-cell lysate}

Whole-cell lysate was extracted from cultured JB6 P+ cells in RIPA lysis buffer (Thermo Fisher Scientific, Waltham, MA, USA) containing the protease inhibitor cocktail (Thermo Fisher Scientific). The supernatant collected to measure the protein concentration was detected by BCA assay (CWBIO, CW0014S).

\section{Western blot analysis}

Forty micrograms of whole-cell lysate were separated by $10 \%$ SDS-PAGE and transferred onto PVDF membranes. Antibodies against IDH1 $(1: 5,000)$, ubiquitin $(1: 2,000)$ and glyceraldehyde 3-phosphate dehydrogenase (GAPDH; 1:5,000) were purchased from Abcam (Cambridge, MA, USA). Antibody against $\beta$-actin $(1: 1,000)$, lactate dehydrogenase (LDH; 1:1,000), glucose transporter 1 (Glut1; $1: 1,000)$, and HIF-1 $\alpha(1: 1,000)$ were purchased from Santa Cruz Biotechnology (Santa Cruz, CA, USA). The membranes were incubated with horseradish peroxidase conjugated anti-rabbit or anti-mouse secondary antibodies $(1: 5,000$, Thermo Fisher Scientific). The blots were detected by Biokit Technology Maxilumin ${ }^{\mathrm{TM}}$-WB Pico Chemiluminescence Substrate reagent (Baizhi, Bejing, China) and exposed to GeneGnome XRQ chemiluminescence film (Gene Company Limited, Winooski, VT, USA).

\section{Proteasome $20 S$ activity assay}

JB6 P+ cells were seeded in a 96-well plate before treated with TPA and/or WA. Proteasome activity was detected using Proteasome 20S Activity Assay Kit (Sigma) according to the manufacturer's instruction. Proteasome assay loading solution was added to each well, and the plate was incubated for $2 \mathrm{~h}$ at $37^{\circ} \mathrm{C}$ protecting from light. The fluorescence densities were monitored at $\lambda \mathrm{ex}=490 \mathrm{~nm}$ and $\lambda \mathrm{em}=525$ $\mathrm{nm}$. The proteasome activity was calculated according to manufacturer's instructions (Gene Company Limited).

\section{Immunoprecipitation}

For identification of ubiquitinated IDH1 protein, $2 \mathrm{mg}$ of whole-cell lysate were freshly prepared. An aliquot of the supernatant was incubated with anti-isocitrate dehydrogenase antibody $(6 \mu \mathrm{L}$ Abs to $2,000 \mu \mathrm{g}$ protein in $500 \mu \mathrm{L}$ cell lysate) overnight at $4{ }^{\circ} \mathrm{C}$ with gentle rotation. 25 $\mu \mathrm{L}$ of pre-cooled protein $\mathrm{G}$ agarose slurry (Cell Signaling Technology, Danvers, MA, USA) was then added and incubated at $4{ }^{\circ} \mathrm{C}$ for 2 hours to capture the immunecomplex, which was then centrifuged at $12,000 \times \mathrm{g}$ at $4{ }^{\circ} \mathrm{C}$ for 2 minutes to collect the conjugates. After washing three times, the conjugates were resuspended with 2 times loading buffer, which were then analyzed by western blot analysis. The membranes were hybridized with primary antibodies followed by incubation with appropriate HRP-conjugated secondary antibodies.

\section{IDH1 siRNA and vector transfections}

Cells were seeded at $2 \times 10^{5}$ per well in 6-well tissue culture plates, and transfection was started when cells became $70-80 \%$ confluent. For each transfection, $2 \mu \mathrm{L}$ of the IDH1 siRNA duplex (Santa Cruz Biotechnology, Santa Cruz, CA, USA) was diluted into $100 \mu \mathrm{L}$ of siRNA transfection medium (Santa Cruz Biotechnology) and labeled as Solution A. Solution B consisted of $2 \mu \mathrm{L}$ of transfection reagent (Santa Cruz Biotechnology) diluted into $100 \mu \mathrm{L}$ of siRNA transfection medium. Solution A and B were mixed gently and incubated for $30 \mathrm{~min}$ at room temperature. Cells were washed once with $2 \mathrm{~mL}$ of siRNA transfection medium. For each transfection, $0.8 \mathrm{~mL}$ of siRNA transfection medium was added to each tube containing the solution $\mathrm{A} / \mathrm{B}$ mixture, mixed and directly added to the washed cells. Six hours later, $2 \mathrm{~mL}$ of $1 \times$ normal growth medium was added to the cells containing the transfection mixture. The cells were incubated for additional $24 \mathrm{~h}$ and subjected to western blot analysis.

For IDH1 overexpression, the cells were washed with PBS and incubated with Opti-MEM medium (Gibco) for 30 min. Five microliter of Lipofectamine 2000 Transfection Reagent (Invitrogen by Thermo Fisher Scientific, Waltham, MA, USA) was diluted with Opti-MEM medium into $125 \mu \mathrm{L}, 2.5 \mu \mathrm{g}$ of pcDNA3.1 vector or pcDNA3.1IDH1 plasmid was diluted with Opti-MEM medium into $125 \mu \mathrm{L}$. The diluted DNA was added to Lipofectamine 2000 reagent (lipo: DNA ratio $=1: 1$ ), followed by mixed gently and incubated for $5 \mathrm{~min}$ at room temperature. For each transfection, the DNA-lipid complex was added to cells. Four hours later, the Opti-MEM containing the transfection complx was replaced by normal growth 
medium. After $48 \mathrm{~h}$, cells were collected and assayed.

\section{Alpha-ketoglutarate quantitative assay}

Cells treated with TPA and/or WA were collected and resuspended with $\alpha-K G$ Buffer. The supernatant was deproteinized by passing through a $10 \mathrm{kD}$ MWCO spin filter (Thermo Scientific Pierce). Two microliters of the whole-cell lysate filtrate was diluted to $50 \mu \mathrm{L}$ with $\alpha-\mathrm{KG}$ Buffer. Alpha-ketoglutarate concentrations were measured using $\alpha$-Ketoglutarate Assay Kit (Sigma) according to the manufacturer's instructions. For each sample, the concentration of $\alpha$-KG was recorded by spectrophotometry at $570 \mathrm{~nm}$, and calculated by the equation provided by the manufacturer (Gene Company Limited).

\section{Mitochondrial complex I activity assay}

The collected cells were lysed by homogenization. The supernatant was collected by centrifugation at $600 \mathrm{~g}$ for 5 min at $4{ }^{\circ} \mathrm{C}$. After then, the precipitate was collected by centrifugation at $11,000 \mathrm{~g}$ for $10 \mathrm{~min}$ at $4{ }^{\circ} \mathrm{C}$. Mitochondrial samples were obtained followed by the precipitate was ultrasonically disrupted on ice. Mitochondrial complex I activity was measured using Mitochondrial Respiratory Chain Complex I Activity Assay Kit (Solarbio, Beijing, China) according to the manufacturer's instruction. The content of complex I was determined at the initiation and 2-min later respectively, which was recorded by spectrophotometry at $340 \mathrm{~nm}$, and calculated by the equation provided by the manufacturer (Gene Company Limited).

\section{LDH activity assay}

Cells were resuspended by LDH assay buffer, and then the supernatant was collected. LDH activity was measured using LDH Activity Assay Kit (Sigma) according to the manufacturer's instructions. The content of LDH was monitored by spectrophotometry at $450 \mathrm{~nm}$, and calculated by the equation provided by the manufacturer (Gene Company Limited).

\section{Mouse vascular endothelial cell growth factor quantitative assay}

Cells gathered were resuspended with PBS, and the process that samples frozen at $-80^{\circ} \mathrm{C}$ for $10 \mathrm{~min}$ was repeated three times. The collected supernatant was the sample solution applied to measure the concentration of vascular endothelial growth factor (VEGF). Mouse VEGF concentration was measured using Mouse VEGF ELISA Kit (Yuanmu Biological Technology, Shanghai, China) according to the manufacturer's instructions (Gene Company Limited).

\section{PHD quantitative assay}

Cells gathered were resuspended and frozen at $-80{ }^{\circ} \mathrm{C}$ for $10 \mathrm{~min}$, which was repeated three times. The collected supernatant was applied to measure the concentration of proline hydroxylase (PHD). PHD concentration was measured using PHD ELISA Kit (Xinyu Biological Technology, Shanghai, China) according to the manufacturer's instructions, which was recorded by spectrophotometry at $450 \mathrm{~nm}$, and calculated by the equation from manufacturer recommended (Gene Company Limited).

\section{Statistical analysis}

One-way analysis of variance (ANOVA) followed by Newman-Keuls post-test was used for multi-group comparisons. All of the experiments have been repeated at least three times. $\mathrm{P}<0.05$ was considered significant.

\section{Results}

\section{Downregulation of IDH1 is predominantly due to tumor promoter TPA-induced protein degradation}

As an important metabolic enzyme, IDH1 reversibly converts isocitrate to $\alpha$-ketoglutarate in cytosol. Previously we have demonstrated that WA inhibits tumor promoter TPA-induced IDH1 inactivation in JB6 P+ cells (Figure S1). To detect if the expression of IDH1 could be regulated in transcription level, the mRNA levels of IDH1 were detected. Data showed that the IDH1 mRNA levels were no significantly changed after TPA and/or WA treatment (Figure 1A). These results suggest that IDH1 might be regulated at translation or post-translation level but not in transcription level.

To further investigate how IDH1 was regulated, a protein synthesis inhibitor cycloheximide (CHX) was employed. JB6 P+ cells were pre-treated with CHX for 4 hours and then treated with TPA and/or WA for 24 hours. The effect of WA on IDH1 protein levels was not changed when cells were pretreated with CHX (Figure 1B,C). Therefore, we speculated WA may be able to stabilize IDH1 at the 
A

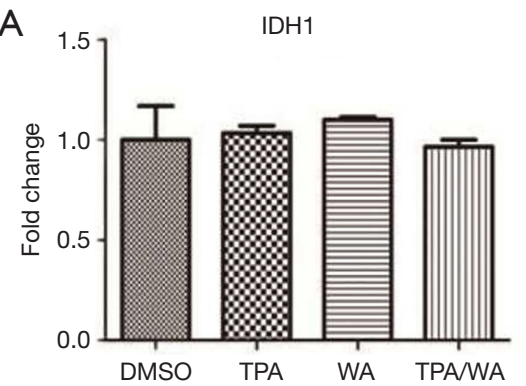

B

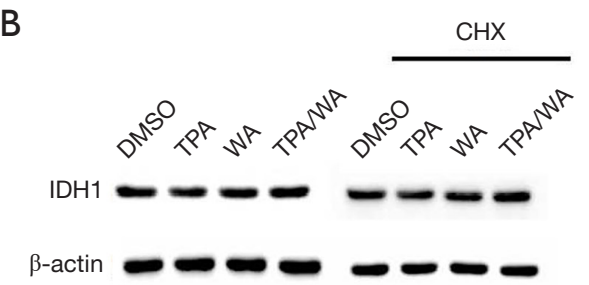

C

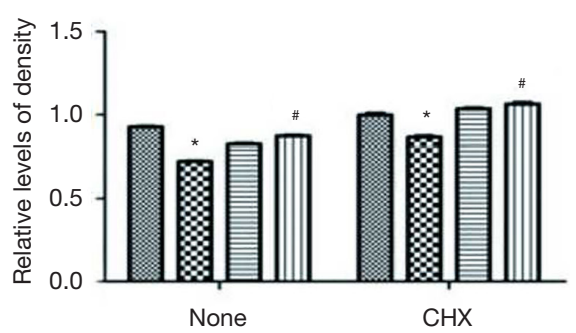

D

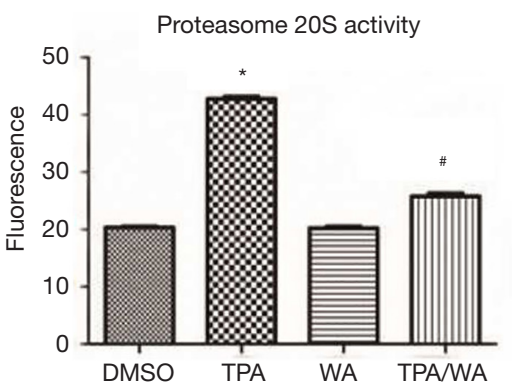

$\mathrm{E}$

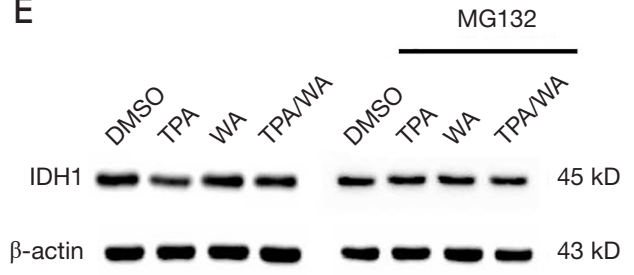

$\mathrm{F}$

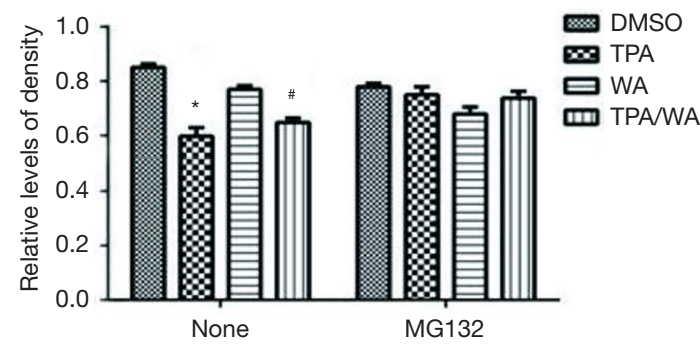

Figure 1 WA inhibited the UPP to stabilize IDH1 protein. (A) JB6 P+ cells were treated with TPA: $5 \mathrm{nM}$; WA: $0.625 \mu$; WA/TPA: cotreatment for $24 \mathrm{~h}$. Measurement of the mRNA levels of IDH1. (B) Cells were pretreated with CHX $(10 \mu \mathrm{g} / \mathrm{mL})$ for $4 \mathrm{~h}$ followed by TPA and/or WA for $24 \mathrm{~h}$. Western blot analysis for IDH1 protein. $\beta$-actin served as the loading control. (C) Densitometric analysis for blots shown in (B). (D) Cells were treated the same as described in (A). Measurement of the proteasome 20S activity. (E) Cells were pretreated with $10 \mu M$ MG132 for $4 \mathrm{~h}$ followed by TPA and/or WA treatments for $24 \mathrm{~h}$. Western blot analysis for IDH1 protein. (F) Densitometric analysis for blots shown in (E). Multi-group analyses were performed using a one-way ANOVA followed by Newman-Keuls post-test. *, TPA compared with the DMSO treatment, $\mathrm{P}<0.05$. ", WA/TPA compared with the TPA treatment, $\mathrm{P}<0.05 . \mathrm{n}=3$ in each group. WA, Withaferin A; UPP, ubiquitin-proteasome pathway; IDH1, isocitrate dehydrogenase 1; TPA, 12-O-tetradecanoylphorbol-13-acetate; CHX, cycloheximide; DMSO, dissolved in dimethyl sulfoxide.

post-translation stage.

The inhibition of target protein degradation through the ubiquitin-proteasome proteolytic pathway which plays an essential role in the protein metabolism is a recently developed approach to cancer treatment. Proteasome $26 \mathrm{~S}$ is the most common form of the proteasome, which contains one $20 \mathrm{~S}$ core particle structure. We first measured the proteasome activity in JB6 $\mathrm{P}+$ cells after treated by TPA and/or WA. TPA-induced significantly increases in proteasome activities (Figure 1D), which contributed to the degradation of protein by activating the proteasome pathway. However, WA attenuated the positive effect of TPA on proteasome (Figure 1D), thereby maintaining protein stability. When the proteasome inhibitor MG132 was applied, the protein levels of IDH1 were restored to normal levels (Figure 1E,F). From the effect of WA/ TPA on JB6 P+ cells decreased by MG132 pretreated, we speculated that WA prevented the downregulation and degradation of IDH1 via inhibiting the UPP. For further investigation, we detected the ubiquitination of IDH1. IDH1 proteins were immuno-precipitated, and detected with an anti-ubiquitin antibody. IDH1 expression levels 

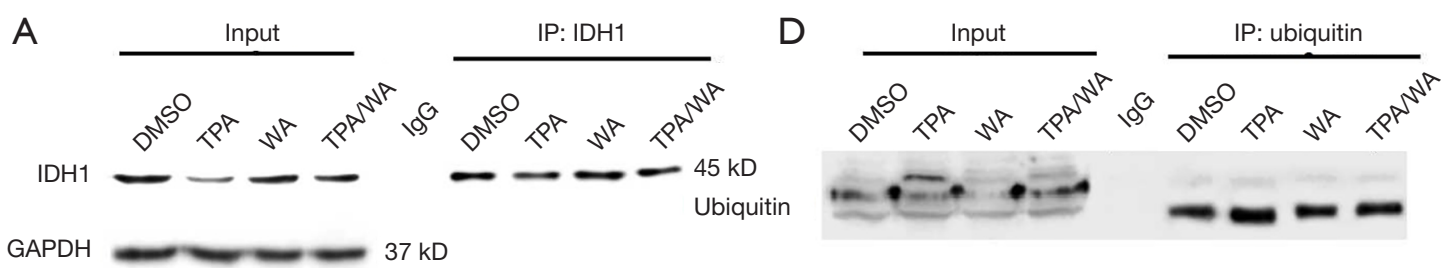

B

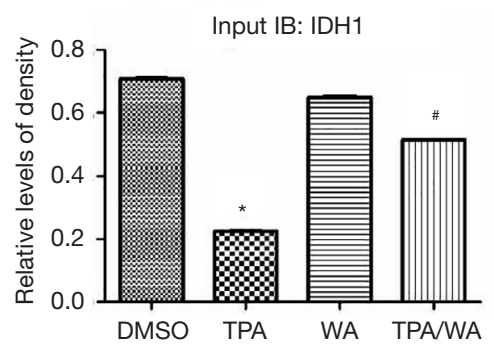

C

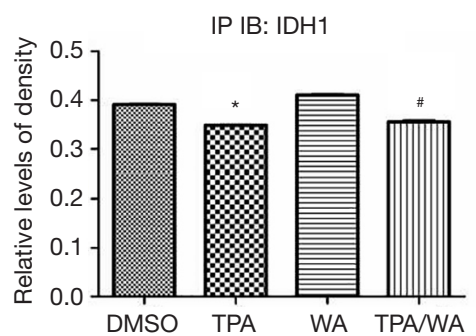

$\mathrm{E}$

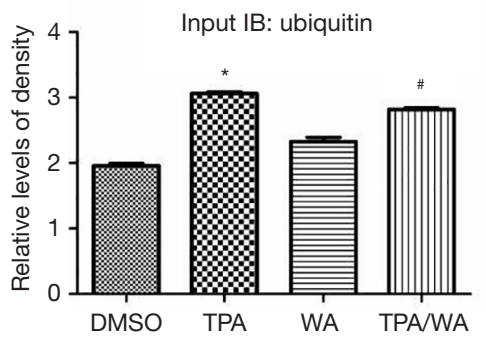

$\mathrm{F}$

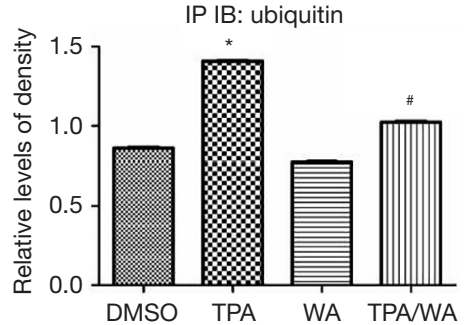

Figure 2 WA inhibited IDH1 ubiquitination. JB6 P+ cells were treated same as before. (A) Western blot analysis for total un-ubiquitinated IDH1. GAPDH served as the loading control. (B) Densitometric analysis for the total un-ubiquitinated IDH1 protein in whole-cell lysis. (C) Densitometric analysis for total un-ubiquitinated IDH1 protein after immunoprecipitation normalized to IgG heavy chain. (D) Western blot analysis for ubiquitinated-IDH1 (Ub-IDH1). (E) Densitometric analysis for the ubiquitinated protein in whole-cell lysis. (F) Densitometric analysis for the ubiquitinated-IDH1 protein after immunoprecipitation. Multi-group analyses were performed using a one-way ANOVA followed by Newman-Keuls post-test. *, TPA compared with the DMSO treatment, $\mathrm{P}<0.05$. ", WA/TPA compared with the TPA treatment, $\mathrm{P}<0.05$. WA, Withaferin A; UPP, ubiquitin-proteasome pathway; IDH1, isocitrate dehydrogenase 1; TPA, 12-O-tetradecanoylphorbol-13acetate; CHX, cycloheximide; DMSO, dissolved in dimethyl sulfoxide.

were significantly increased after WA treatment in contrast with that TPA treatment both in the whole-cell lysate and the lysate followed by immunoprecipitation (Figure $2 A, B, C$ ). However, increased ubiquitinated IDH1 was observed after TPA treatment before and after immunoprecipitation, indicating that TPA induced the ubiquitination and degradation of IDH1 protein (Figure 2D,E,F). In contrast, WA treatment reduced the ubiquitinated IDH1 and proteasome activity. These results further demonstrate that WA could stabilize IDH1 by inhibiting the UPP.

\section{IDH1, as a possible tumor suppressor, inbibits aerobic glycolysis and maintains mitochondrial functions}

To explore how altered levels of IDH1 affects cellular metabolism, we either overexpressed or knocked down IDH1 in JB6 P+ cells. At first, JB6 P+ cells were transfected with either control siRNA or siRNA to IDH1, and the transfection efficiency was monitored by the IDH1 expression (Figure $S 2 A, B$ ). Similarly, cells were transfected with the pcDNA3.1 or pcDNA3.1IDH1 vector, and the transfection efficiency was also detected by the IDH1 expression (Figure $S 2 C, D$ ). The concentration of $\alpha-K G$, the product of IDH1, was detected in these transfected cells. Alpha ketoglutarate levels were significantly increased in pcDNA3.1-IDH1 compared with siRNA-IDH1-transfected cells (Figure 3A). Next, we measured mitochondrial complex I activity, a marker for mitochondrial functions. The complex I activity was significantly increased in pcDNA3.1-IDH1- 
transfected, but slightly decreased in siRNA-IDH1transfected cells (Figure 3B). The collected data mirrored that IDH1 functioned in protecting the mitochondria from dysfunction. To observe the impact of IDH1 on glycolysis in carcinogenesis, the expression levels of $\mathrm{LDH}$ which is the essential enzyme involved in glycolysis, were detected. WA suppressed the TPA-induced upregulation of LDH expression (Figure 3C,D). WA as an anti-tumor agent might block the tumorigenesis on account of reducing the energy supply provided by glycolysis. Overexpression of IDH1 also inhibited LDH expression, which was reversed by knockdown of IDH1 (Figure 3E,F). In addition, the LDH activity was determined by using the same samples collected from JB6 P+ cells which were transfected with pcDNA3.1IDH1. IDH1 overexpression reduced the activity of LDH (Figure 3G). These observations indicate that IDH1 might ward off tumorigenesis just as WA, which was mediated by the inhibition on glycolysis.

\section{WA suppresses the HIF-1a pathway}

Hypoxia inducible factor- $1 \alpha$ (HIF-1 $\alpha$ ) is indispensable in hypoxia, which regulates the biological metabolism of cancer cells to maintain their proliferation. As a cofactor, $\alpha-K G$ produced from IDH1 regulates the activity of PHD, and PHD inhibits HIF- $1 \alpha$ activity. Here we measured the expression of HIF-1 $\alpha$ targeted genes, including glucose transport protein (Glut) and VEGF. The expression levels of Glut1 and HIF-1 $\alpha$ were increased after TPA treatment, which were inhibited by WA (Figure 4A,B). IDH1 overexpression also inhibited the expression of Glut and HIF-1 $\alpha$, but the downregulation of IDH1 enhanced its expression (Figure 4C,D). It was consistent that WA and IDH1 overexpression had the similar impact on the downstream of HIF-1 $\alpha$. Then, the results revealed that WA decreased the levels of VEGF (upper panel), but increased the levels of PHD (lower panel) (Figure 4E). The reduced levels of VEGF by either WA or IDH1 overexpression (Figure $4 F$ ) were consistent with the results of Glut1 and HIF-1 $\alpha$, which demonstrated that WA might be an inhibition agent to HIF-1 $\alpha$ pathway. The finding that increased level of PHD coincided with the accumulation of $\alpha-K G$ when IDH1 was overexpressed also further supported the connection between PHD and IDH1. Collectively, our results indicate that WA stabilizes IDH1 during tumor promotion, and maintains the activity of PHD which inhibits the activation of HIF- $1 \alpha$, serving as an important mechanism of chemoprevention.

\section{Discussion}

IDH1 converts isocitrate to $\alpha-\mathrm{KG}$ in the cytoplasm, which activates dioxygenases. Another metabolic enzyme glutamate dehydrogenase (GDH) also converts glutamate to $\alpha-K G(24)$. Our early studies have demonstrated neither TPA nor WA treatment alters GDH activity (16), suggesting that the changes in the $\alpha-K G$ levels mainly come from IDH. Alpha-KG shuttles between the cytosol and mitochondria so that IDH1 is indirectly involved in the tri-carboxylic acid cycle (TCA) (25). Our data have demonstrated that elevated levels of IDH1 increases whereas reduced levels of IDH1 decreases mitochondrial complex I activity, suggesting that IDH1 could maintain the normal mitochondrial functions. Tumorigenesis is often associated with a metabolic shift. Cancer cells predominantly produce energy by a high rate of glycolysis followed by lactic acid fermentation even in the presence of oxygen, known as the "Warburg Effect" (1). Our data show that WA could significantly block the expression of LDH, and overexpression of IDH1 also inhibits both the expression and activity levels of LDH, suggesting a possible mechanism that IDH1 suppresses tumor promotion via inhibiting glycolysis.

WA is a bioactive compound derived from Withania somnifera, which has been used in clinic for the treatment of nervous and sexual disorders (8). Our preliminary studies have shown that WA inhibited skin tumorigenesis both in vitro and in vivo combined with the overexpression and increased enzyme activity of IDH1 $(16,26)$. Nevertheless, how WA acts as a chemopreventive agent mediated by IDH1 is unclear. It is timely and significant to understand the mechanism of WA in chemoprevention. Our early studies show that WA could block tumor promoter TPAinduced inactivation of IDH1 during tumor promotion (16). As a target gene of nuclear factor erythroid 2-related factor 2 (Nrf2), the promoter of IDH1 includes the antioxidant response element (ARE) which is the binding site of $\mathrm{Nrf} 2$ $(27,28)$. We measured the mRNA level of Nrf2 (Figure S3), and results show that TPA treatment suppresses the mRNA levels of Nrf2, which is blocked by WA. However, the mRNA levels of IDH1 are not significantly changed during these treatments (Figure 1A), suggesting the effects on IDH1 are not due to its gene transcription. When applying the protein synthesis inhibitor CHX, the protein levels of IDH1 do not show significant differences between different treatments, suggesting the effects on IDH1 are not due to protein synthesis or the modification of post-translation. Therefore, we focus on whether WA stabilizes IDH1 


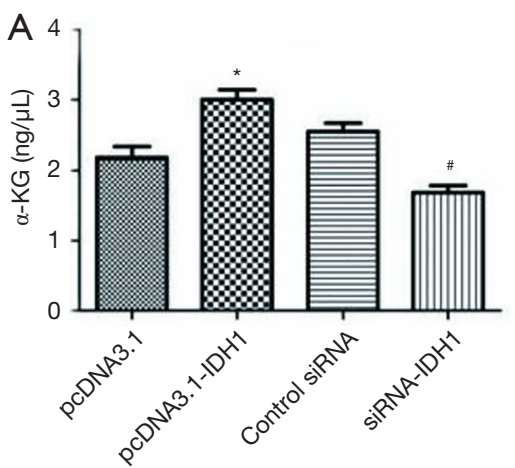

B
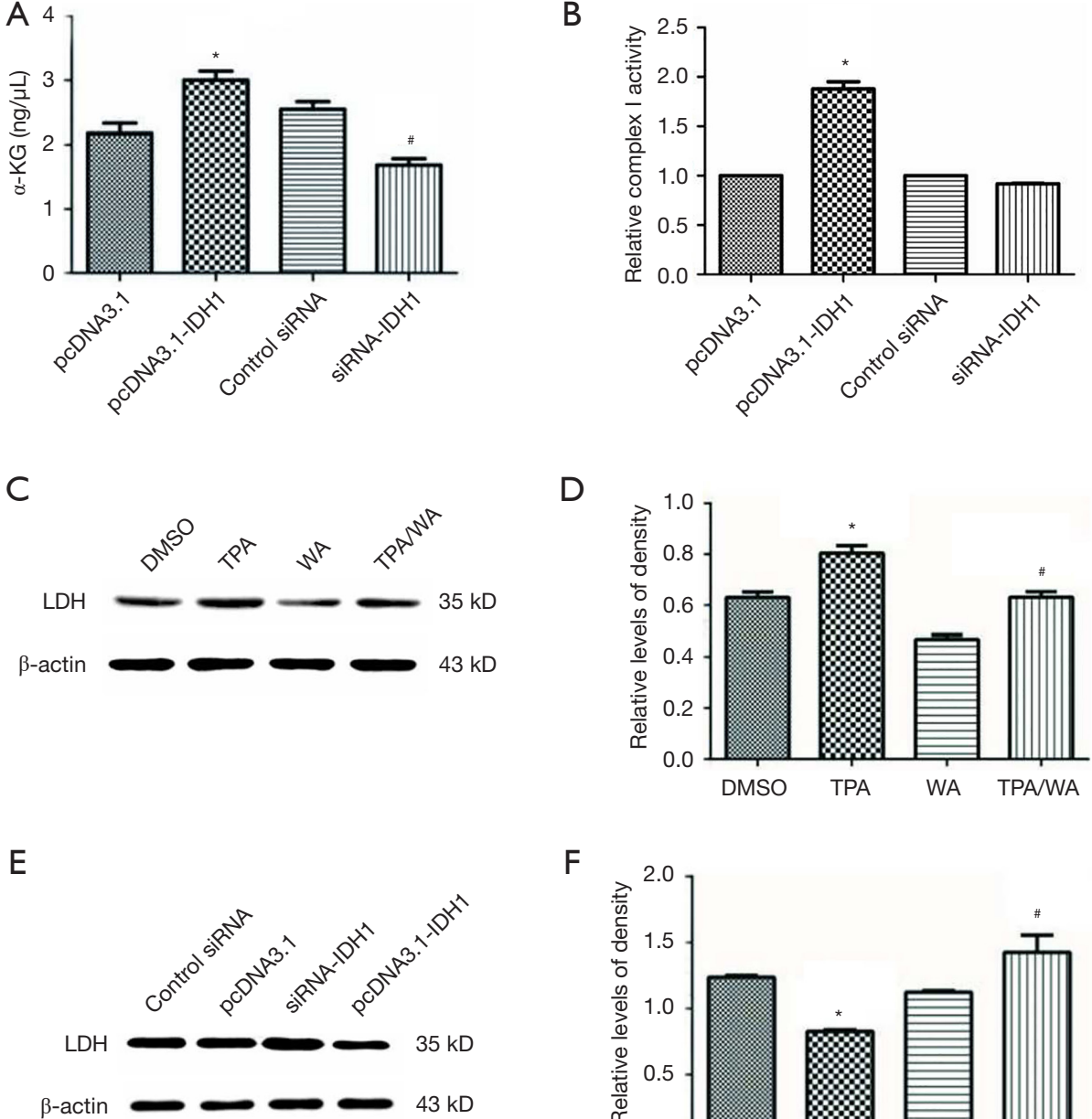

$\mathrm{F}$

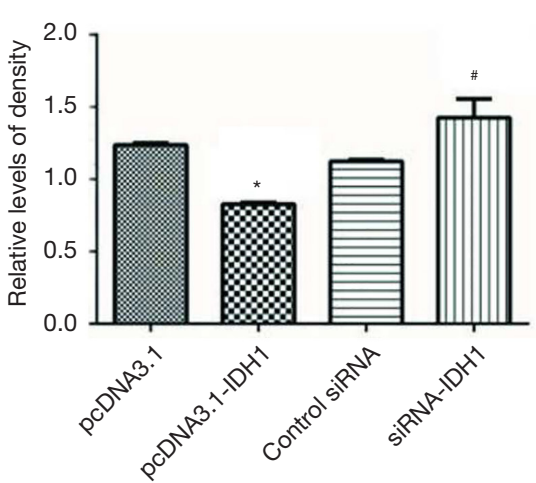

G

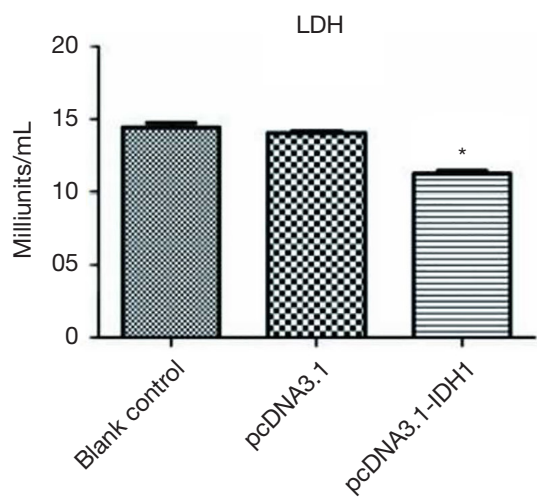

Figure 3 The effect of IDH1 on LDH and complex I activities. JB6 P+ cells were transfected with pcDNA3.1-IDH1 or siRNA-IDH1 for 6 h. (A) The concentrations of $\alpha$-KG. (B) The activity of mitochondrial complex I. (C) JB6 P+ cells were treated same as before. Western blot analysis for endogenous LDH. (D) Densitometric analysis for blots shown in (C). (E) Western blot analysis for endogenous LDH. (F) Densitometric analysis for blots shown in (E). (G) Detection of LDH activity. Multi-group analyses were performed using a oneway ANOVA followed by Newman-Keuls post-test. *, pcDNA3.1-IDH1 compared with the pcDNA3.1 treatment, P<0.05. *, siRNAIDH1 compared with the pcDNA3.1-IDH1 treatment, $\mathrm{P}<0.05$. WA, Withaferin A; UPP, ubiquitin-proteasome pathway; IDH1, isocitrate dehydrogenase 1; TPA, 12-O-tetradecanoylphorbol-13-acetate; CHX, cycloheximide; DMSO, dissolved in dimethyl sulfoxide. 
A

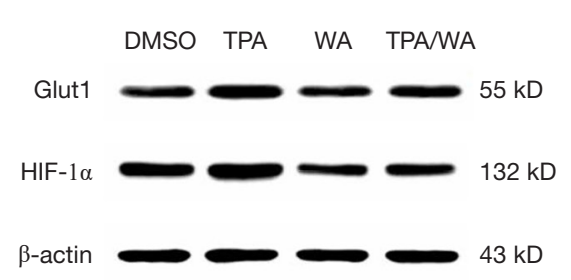

C

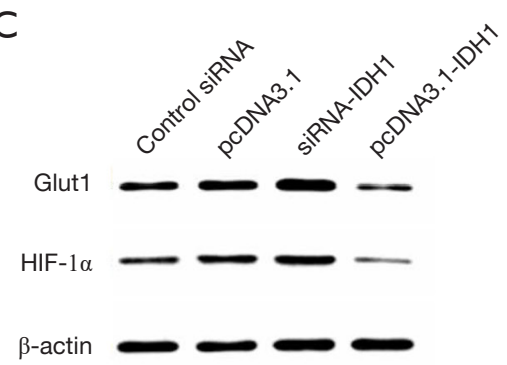

E
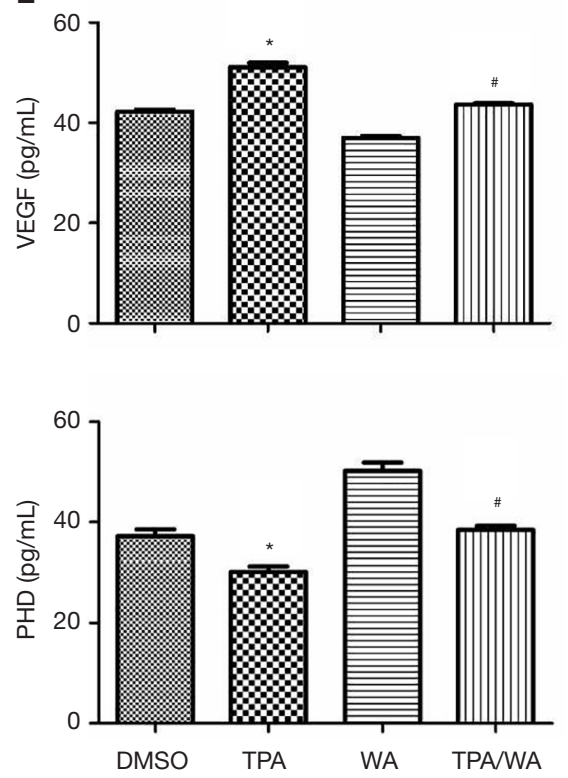

B

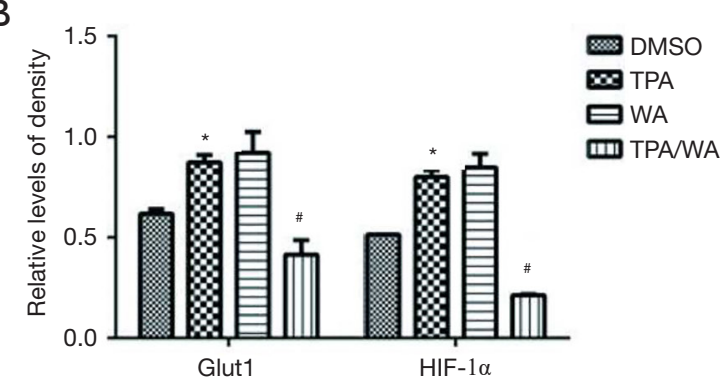

D

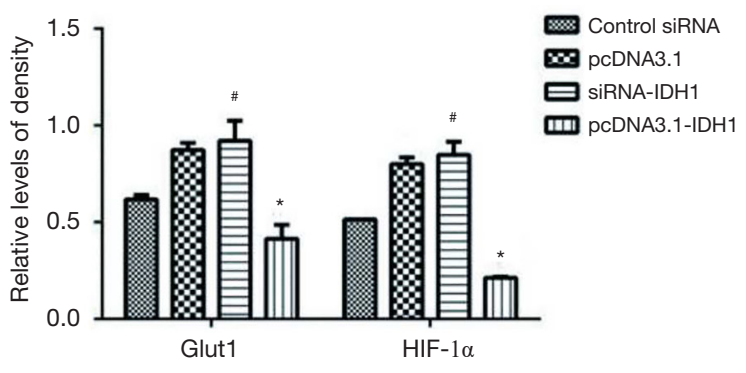

$\mathrm{F}$
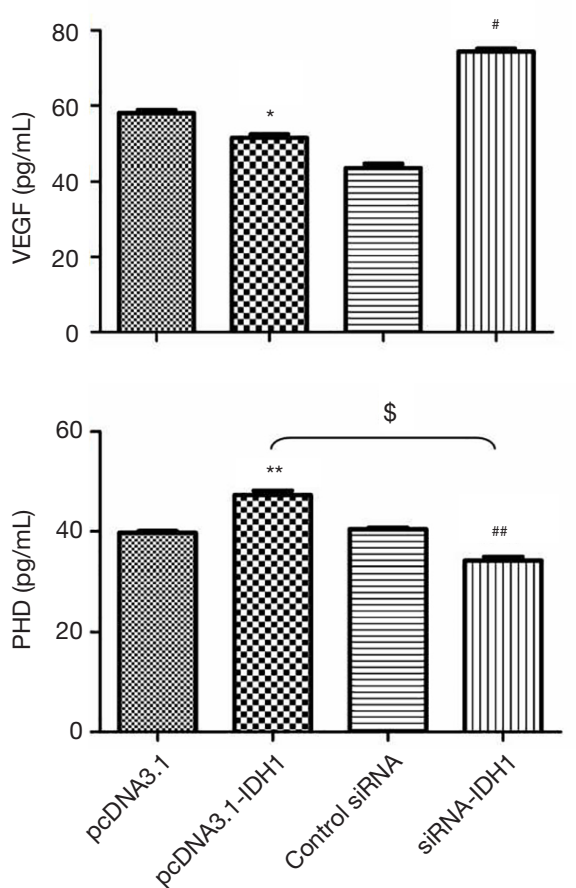

Figure 4 Both WA and IDH1 inhibited the HIF-1 $\alpha$ signaling. Western blot analysis for HIF-1 $\alpha$ and Glut1 after WA treatment (A) or IDH1 vector transfection (C) in JB6 P+ cells. (B) Densitometric analysis for blots shown in (A). (D) Densitometric analysis for blots shown in (C). Detection of the activity of VEGF (upper panel) and PHD (lower panel) after WA treatment (E) or IDH1 vector transfection (F). Multi-group analyses were performed using a one-way ANOVA followed by Newman-Keuls post-test. *, TPA compared with the DMSO treatment, $\mathrm{P}<0.05$. *, WA/TPA compared with the TPA treatment, $\mathrm{P}<0.05$. **, pcDNA3.1-IDH1 compared with pcDNA3.1 treatment, $\mathrm{P}<0.05$. , siRNA-IDH1 compared with the control siRNA treatment, $\mathrm{P}<0.05$. \$, siRNA-IDH1 compared with the pcDNA3.1-IDH1 treatment, $\mathrm{P}<0.05 . \mathrm{n}=3$ in each group. WA, Withaferin A; UPP, ubiquitin-proteasome pathway; IDH1, isocitrate dehydrogenase 1; TPA, 12-O-tetradecanoylphorbol-13-acetate; CHX, cycloheximide; DMSO, dissolved in dimethyl sulfoxide. 
during post-translation stage. There are two major and fundamentally different mechanisms by which cells degrade proteins, the lysosome and the proteasome $(29,30)$. The UPP is a principal and highly effective protein-degradation pathway in the eukaryotic cells (31), which regulates a wild range of cellular biology including cell growth (32), cell signaling (33), cell immunity (34), cell differentiation (35), and other important cellular physiological processes. The disturbance in the ubiquitination process may lead to tumor promotion (36). WA has showed anti-angiogenesis pharmacological activities through targeting the UPP (37). Therefore, we measure the proteasome activity and in deed, WA suppresses TPA induced proteasome activity. Followed by employing the proteasome inhibitor MG132, WA prevented the downregulation and degradation of IDH1 induced by tumor promoter TPA via inhibiting the UPP. It is further demonstrated by results showing that WA blocks TPA-induced ubiquitination of IDH1, which is consistent with the function of WA as a proteasome inhibitor (38). Since the modification of IDH1 by ubiquitin is of great significance in regulating its degradation and activity, thereby furthermore generates biological effect, what kind of ubiquitinations occurs in IDH1? Which sites of IDH1 are modified by ubiquitin? The studies on the detection of ubiquitination sites are further conducting to explore the detail mechanism.

As a downstream molecular of $\alpha-\mathrm{KG}$, PHD inhibits the HIF- $1 \alpha$-induced signal pathway involved in tumorigenesis $(39,40)$. Our data are showing that PHD activity is induced by WA treatment, as well IDH1 overexpression, establishing a link WA to HIF-1 $\alpha$. It is further supported by the data that WA blocks TPA-induced activation of Glut 1 and VEGF, both the target molecules of HIF-1 $\alpha$ and contributing to tumorigenesis $(41,42)$.

\section{Conclusions}

Taken together, our present study has demonstrated a novel mechanism of how WA, as a potential chemopreventive agent, inhibits tumor promotion, starting from stabilizing IDH1 to inactivating HIF-1 $\alpha$ signaling. These results uncover the mechanism of tumor suppressor gene IDH1 as a promising molecular target for chemoprevention, and WA might provide a guide for the discovery of chemopreventive chemicals.

\section{Acknowledgments}

Funding: This work was supported by The National
Natural Science Foundation of China (No. 81502477); Hebei Provincial Natural Science Foundation of China (No, H2019201084); Research Fund for Introduced Talents of Hebei University (No. 2015-307), and Program (LBH-Q17177) and Grants from Committee of Education Science Foundation of Heilongjiang Province (2017-QYKYYWF-0754).

\section{Footnote}

Conflicts of Interest: The authors have completed the ICMJE uniform disclosure form (available at http://dx.doi. org/10.21037/tcr.2019.09.57). The authors have no conflicts of interest to declare.

Ethical Statement: The authors are accountable for all aspects of the work in ensuring that questions related to the accuracy or integrity of any part of the work are appropriately investigated and resolved. The research was conducted at the molecular and cellular levels, and didn't employ the animal model. The ethical statement was not required.

Open Access Statement: This is an Open Access article distributed in accordance with the Creative Commons Attribution-NonCommercial-NoDerivs 4.0 International License (CC BY-NC-ND 4.0), which permits the noncommercial replication and distribution of the article with the strict proviso that no changes or edits are made and the original work is properly cited (including links to both the formal publication through the relevant DOI and the license). See: https://creativecommons.org/licenses/by-nc-nd/4.0/.

\section{References}

1. Warburg O. On the origin of cancer cells. Science 1956;123:309-14.

2. Vander Heiden MG, Cantley LC, Thompson CB. Understanding the Warburg effect: the metabolic requirements of cell proliferation. Science 2009;324:1029-33.

3. Rohle D, Popovici-Muller J, Palaskas N, et al. An inhibitor of mutant IDH1 delays growth and promotes differentiation of glioma cells. Science 2013;340:626-30.

4. Figueroa ME, Abdel-Wahab O, Lu C, et al. Leukemic IDH1 and IDH2 mutations result in a hypermethylation phenotype, disrupt TET2 function, and impair hematopoietic differentiation. Cancer Cell 2010;18:553-67. 
5. Yang Z, Jiang B, Wang Y, et al. 2-HG Inhibits Necroptosis by Stimulating DNMT1-Dependent Hypermethylation of the RIP3 Promoter. Cell Rep 2017;19:1846-57.

6. Dang L, White DW, Gross S, et al. Cancer-associated IDH1 mutations produce 2-hydroxyglutarate. Nature 2009;462:739-44.

7. Laba P, Wang J, Zhang J. Low level of isocitrate dehydrogenase 1 predicts unfavorable postoperative outcomes in patients with clear cell renal cell carcinoma. BMC Cancer 2018;18:852.

8. Chowdhury K, Neogy RK. Mode of action of Withaferin A and Withanolide D. Biochem Pharmacol 1975;24:919-20.

9. Liu X, Chen L, Liang T, et al. Withaferin A induces mitochondrial-dependent apoptosis in non-small cell lung cancer cells via generation of reactive oxygen species. J BUON 2017;22:244-50.

10. Zhang HL, Zhang H. Withaferin-A Induces Apoptosis in Osteosarcoma U2OS Cell Line via Generation of ROS and Disruption of Mitochondrial Membrane Potential. Pharmacogn Mag 2017;13:523-27.

11. Ndlovu MN, Van Lint C, Van Wesemael K, et al. Hyperactivated NF-\{kappa\}B and AP-1 transcription factors promote highly accessible chromatin and constitutive transcription across the interleukin- 6 gene promoter in metastatic breast cancer cells. Mol Cell Biol 2009;29:5488-504.

12. Srinivasan S, Ranga RS, Burikhanov R, et al. Par-4dependent apoptosis by the dietary compounwithaferin A in prostate cancer cells. Cancer Res 2007;67:246-53.

13. Koduru S, Kumar R, Srinivasan S, et al. Notch-1 inhibition by Withaferin-A: a therapeutic target against colon carcinogenesis. Mol Cancer Ther 2010;9:202-10.

14. Yu Y, Hamza A, Zhang T, et al. Withaferin A targets heat shock protein 90 in pancreatic cancercells. Biochem Pharmacol 2010;79:542-51.

15. Walker K, Padhiar M. AACR-NCI-EORTC--21st International Symposium. Molecular targets and cancer therapeutics--Part 1. IDrugs 2010;13:7-9.

16. Li W, Zhao Y. Withaferin A suppresses tumor promoter 12-O-tetradecanoylphorbol 13-acetate-induced decreases in isocitrate dehydrogenase 1 activity and mitochondrial function in skin epidermal JB6 cells. Cancer Sci 2013;104:143-8.

17. Khedgikar V, Kushwaha P, Gautam J, et al. Withaferin A: a proteasomal inhibitor promotes healing after injury and exerts anabolic effect on osteoporotic bone. Cell Death Dis 2013;4:e778.

18. Zhang X, Timmermann B, Samadi AK, et al. Withaferin a induces proteasome-dependent degradation of breast cancer susceptibility gene 1 and heat shock factor 1 proteins in breast cancer cells. ISRN Biochem 2012;2012:707586.

19. Liu J, Shaik S, Dai X, et al. Targeting the ubiquitin pathway for cancer treatment. Biochim Biophys Acta 2015;1855:50-60.

20. Rossi M, Aqeilan RI, Neale M, et al. The E3 ubiquitin ligase Itch controls the protein stability of p63. Proc Natl Acad Sci U S A 2006;103:12753-8.

21. Wang X, Trotman LC, Koppie T, et al. NEDD4-1 is a proto-oncogenic ubiquitin ligase for PTEN. Cell 2007;128:129-39.

22. Grover A, Shandilya A, Bisaria VS, et al. Probing the anticancer mechanism of prospective herbal drug Withaferin A on mammals: a case study on human and bovine proteasomes. BMC Genomics 2010;11 Suppl 4:S15.

23. Yang H, Shi G, Dou QP. The tumor proteasome is a primary target for the natural anticancer compound Withaferin A isolated from "Indian winter cherry". Mol Pharmacol 2007;71:426-37.

24. Lu Z, Scott I, Webster BR, et al. The emerging characterization of lysine residue deacetylation on the modulation of mitochondrial function and cardiovascular biology. Circ Res 2009;105:830-41.

25. Frezza C, Tennant DA, Gottlieb E. IDH1 mutations in gliomas: when an enzyme loses its grip. Cancer Cell 2010;17:7-9.

26. Li W, Zhang C, Du H, et al. Withaferin A suppresses the up-regulation of acetyl-coA carboxylase 1 and skin tumor formation in a skin carcinogenesis mouse model. Mol Carcinog 2016;55:1739-46.

27. Xu C, Huang MT, Shen G, et al. Inhibition of 7,12-dimethylbenz(a)anthracene-induced skin tumorigenesis in C57BL/6 mice by sulforaphane is mediated by nuclear factor E2-related factor 2. Cancer Res 2006;66:8293-6.

28. Chen C, Kong AN. Dietary chemopreventive compounds and ARE/EpRE signaling. Free Radic Biol Med 2004;36:1505-16.

29. Mizushima N, Levine B, Cuervo AM, et al. Autophagy fights disease through cellular self-digestion. Nature 2008;451:1069-75.

30. Reinstein E, Ciechanover A. Narrative review: protein degradation and human diseases: the ubiquitin connection. Ann Intern Med 2006;145:676-84.

31. Hershko A, Ciechanover A. The ubiquitin system. Annu Rev Biochem 1998;67:425-79. 
32. Benanti JA. Coordination of cell growth and division by the ubiquitin-proteasome system. Semin Cell Dev Biol 2012;23:492-98.

33. Willis MS, Townley-Tilson WH, Kang EY, et al. Sent to destroy: the ubiquitin proteasome system regulates cell signaling and protein quality control in cardiovascular development and disease. Circ Res 2010;106:463-78.

34. Wang J, Maldonado MA. The ubiquitin-proteasome system and its role in inflammatory and autoimmune diseases. Cell Mol Immunol 2006;3:255-61.

35. Naujokat C, Saric T. Concise review: role and function of the ubiquitin-proteasome system in mammalian stem and progenitor cells. Stem Cells 2007;25:2408-18.

36. Chen X, Shen J, Li X, et al. Rlim, an E3 ubiquitin ligase, influences the stability of Stathmin protein in human osteosarcoma cells. Cell Signal 2014;26:1532-38.

37. Mohan R, Hammers HJ, Bargagna-Mohan P, et al. Withaferin $\mathrm{A}$ is a potent inhibitor of angiogenesis. Angiogenesis 2004;7:115-22.

Cite this article as: $\mathrm{Xu} \mathrm{K}$, Zhang $\mathrm{C}$, Li Y, Xi X, Zheng L, Meng M, Liu T, Zhao Y, Li W. Withaferin A suppresses skin tumor promotion by inhibiting proteasome-dependent isocitrate dehydrogenase 1 degradation. Transl Cancer Res 2019;8(6):2449-2460. doi: 10.21037/tcr.2019.09.57
38. Yang H, Wang Y, Cheryan VT, et al. Withaferin A inhibits the proteasome activity in mesothelioma in vitro and in vivo. PLoS One 2012; 7:e41214.

39. Mimeault M, Batra SK. Hypoxia-inducing factors as master regulators of stemness properties and altered metabolism of cancer- and metastasis-initiating cells. J Cell Mol Med 2013;17:30-54.

40. Partch CL, Gardner KH. Coactivators necessary for transcriptional output of the hypoxia inducible factor, HIF, are directly recruited by ARNT PAS-B. Proc Natl Acad Sci U S A 2011;108:7739-44.

41. Armitage EG, Kotze HL, Allwood JW, et al. Metabolic profiling reveals potential metabolic markers associated with Hypoxia Inducible Factor-mediated signalling in hypoxic cancer cells. Sci Rep 2015;5:15649.

42. Lee SY, Kim HJ, Oh SC, et al. Genipin inhibits the invasion and migration of colon cancer cells by the suppression of HIF-1alpha accumulation and VEGF expression. Food Chem Toxicol 2018;116:70-6. 
A

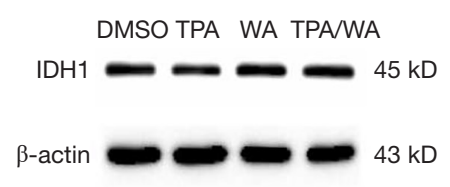

B

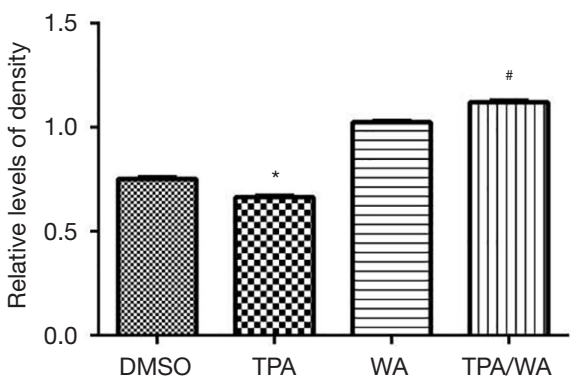

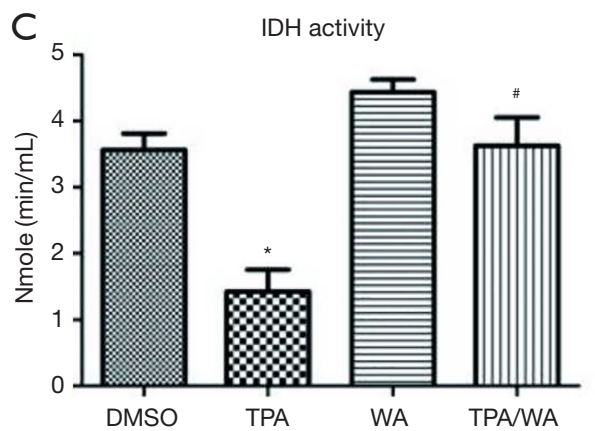

Figure S1 The effect of WA or TPA on IDH1 protein expression and activity. WA inhibited tumor promoter TPA-induced both IDH1 protein (A) and activity (C) downregulation in JB6 P+ cells. (B) Densitometric analysis for blots shown in (A). Multi-group analyses were performed using a one-way ANOVA followed by Newman-Keuls post-test. *, TPA compared with the DMSO treatment, P<0.05. *, WA/ TPA compared with the TPA treatment, $\mathrm{P}<0.05 . \mathrm{n}=3$ in each group. WA, Withaferin A; UPP, ubiquitin-proteasome pathway; IDH1, isocitrate dehydrogenase 1; TPA, 12-O-tetradecanoylphorbol-13-acetate; CHX, cycloheximide; DMSO, dissolved in dimethyl sulfoxide.

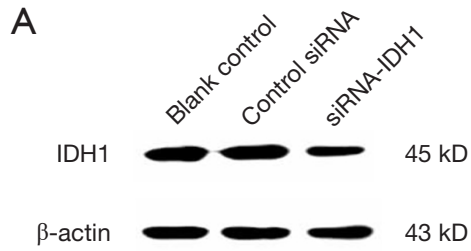

C

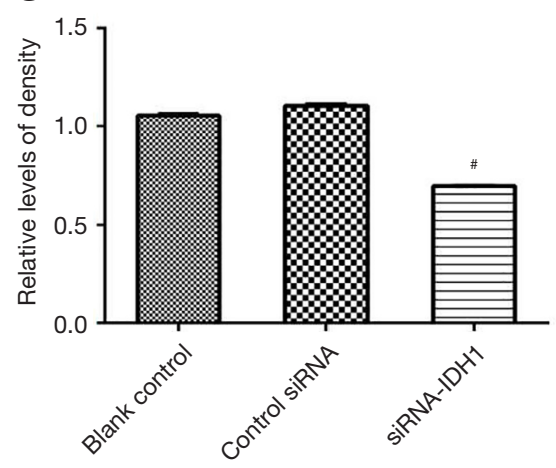

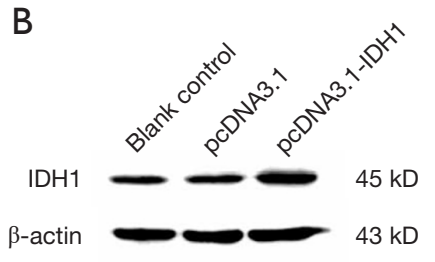

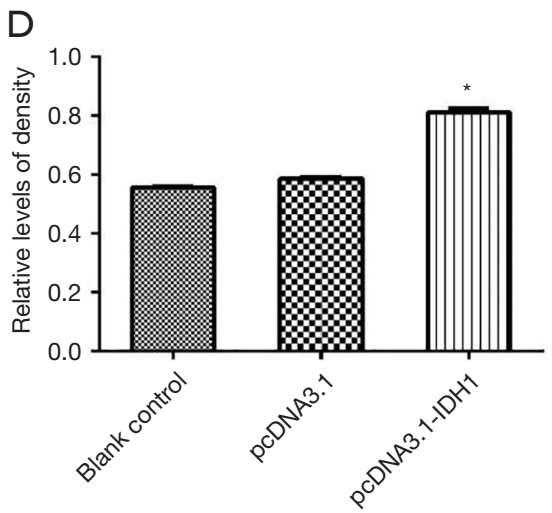

Figure S2 The corresponding data about the overexpression and knockdown of IDH1. JB6 P+ cells were transfected with control siRNA or siRNA-IDH1(A) and pcDNA3.1 or pcDNA3.1-IDH1(C) for 6 h. (B) Densitometric analysis for blots shown in (A). (D) Densitometric analysis for blots shown in (C). Western blot analysis for IDH1. *, TPA compared with the DMSO treatment, P<0.05; \#, WA/TPA compared with the TPA treatment, $\mathrm{P}<0.05$. WA, Withaferin A; UPP, ubiquitin-proteasome pathway; IDH1, isocitrate dehydrogenase 1; TPA, 12-O-tetradecanoylphorbol-13-acetate; CHX, cycloheximide; DMSO, dissolved in dimethyl sulfoxide. 


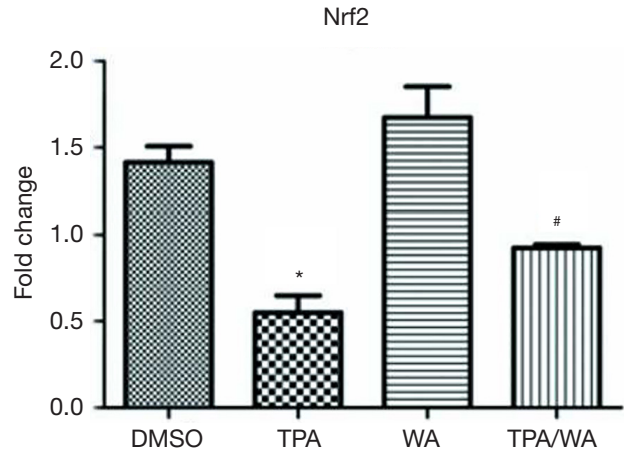

Figure S3 WA suppressed TPA-induced downregulation of the mRNA levels of Nrf2. JB6 P+ cells were treated same as before. Measurement of the mRNA levels of Nrf2. Multi-group analyses were performed using a one-way ANOVA followed by Newman-Keuls post-test. *, TPA compared with the DMSO treatment, $\mathrm{P}<0.05$. *, WA/TPA compared with the TPA treatment, $\mathrm{P}<0.05$. $\mathrm{n}=3$ in each group. WA, Withaferin A; UPP, ubiquitin-proteasome pathway; IDH1, isocitrate dehydrogenase 1; TPA, 12-O-tetradecanoylphorbol-13-acetate; CHX, cycloheximide; DMSO, dissolved in dimethyl sulfoxide. 